Filmes comestíveis de blendas políméricas de quitosana, pectina e fécula de mandioca

\title{
Edible films from polyneric blends of chitosan, pectin and cassava starch
}

\section{Tamara Lorena Eufrazio da Costa ${ }^{1}$; Ricardo Henrique de Lima Leite ${ }^{2}$; Edna Maria Mendes Aroucha ${ }^{3}$ Francisco Klebson Gomes dos Santos ${ }^{4}$}

${ }^{1}$ Mestranda do Programa de Pós-Graduação em Ciência e Engenharia de Materiais, da Universidade Federal Rural do Semi-Árido, Mossoró, Rio Grande do Norte; loreninha_0403@ hotmail.com; 2Professor da Universidade Federal Rural do Semi-Árido, Mossoró, RN, +558433178200, ricardoleite@ufersa.edu.br; ${ }^{3}$ Professora da Universidade Federal Rural do Semi-Árido, Mossoró, RN, aroucha@ufersa.edu.br; ${ }^{4}$ Professor da Universidade Federal Rural do Semi-Árido, Mossoró, RN, klebson@ufersa.edu.br

\section{A R T I G O \\ Recebido: 04/07/2019 \\ Aprovado: 24/09/2020}

\section{Palavras-chave:}

Filmes Comestíveis

Propriedades de barreira

Vida de prateleira

Key words:

Edible Films

Barrier properties

Shelf life

\begin{abstract}
R E S U M O
Os materiais poliméricos não biodegradáveis, tem se tornado um problema devido ao longo tempo de degradação destes no meio ambiente. Assim, estudos demonstram interesse em relação ao desenvolvimento de filmes poliméricos e misturas destes como materiais funcionais nas mais diversas áreas, especialmente devido a suas características como biocompatibilidade, biodegradabilidade e não-toxicidade. Logo, objetivou-se desenvolver e caracterizar filmes comestíveis produzidos por blendas poliméricas, compostas por polissacarídeos naturais, visando sua utilização como recobrimentos comestíveis para frutos. Foram desenvolvidos sete filmes poliméricos pela técnica casting: filme 1 (Quitosana), filme 2 (Pectina), filme 3 (Fécula de Mandioca), filme 4 (Quitosana + Pectina), filme 5 (Quitosana + Fécula de Mandioca), filme 6 (Pectina + Fécula de Mandioca) e filme 7 (Quitosana + Pectina + Fécula de Mandioca). Todos apresentaram superfícies homogêneas e sem separação de fases. Em relação às propriedades óticas, todos obtiveram alta luminosidade, sendo que os mais opacos foram os de quitosana, apresentando $L^{*} 77,40 \pm 0,28$ (4). Quanto as propriedades de barreira, os valores de solubilidade foram menores nos que continham quitosana devido a sua insolubilidade em água, sendo $22 \%$ para o 1 e $18 \%$ para o 5 . Os filmes formados pelas blendas proporcionaram redução da taxa de permeação de água sem comprometer suas características, como os filmes 4 e 5 , com taxa de $14,15 \mathrm{~g} \cdot \mathrm{m}^{-2} \cdot \mathrm{h}^{-1}$ e $20,43 \mathrm{~g} \cdot \mathrm{m}^{-2} \cdot \mathrm{h}^{-1}$, respectivamente.
\end{abstract}

\section{INTRODUÇÃO}

Filmes comestíveis produzidos por polímeros podem ser uma alternativa eficiente para o prolongamento da vida útil dos alimentos (FAKHOURI et al., 2015), por outro lado, os mesmos são bastantes hidrofílicos e tendem a se desestruturar

\section{A B S T R A C T}

Non-biodegradable polymeric materials have become a problem due to their long degradation time in the environment. Thus, studies show interest in the development of polymeric films and mixtures of these as functional materials in the most diverse areas, especially due to their characteristics such as biocompatibility, biodegradability and non-toxicity. Therefore, the objective was to develop and characterize edible films produced by polymeric blends, composed of natural polysaccharides, aiming at their use as edible coatings for fruits. Seven polymeric films were developed using the casting technique: film 1 (Chitosan), film 2 (Pectin), film 3 (Cassava Starch), film 4 (Chitosan + Pectin), film 5 (Chitosan + Cassava Starch), film 6 (Pectin + Cassava Starch) and film 7 (Chitosan + Pectin + Cassava Starch). All presented homogeneous surfaces and without phase separation. Regarding optical properties, all obtained high luminosity, the most opaque being those of chitosan, presenting L * 77.40 \pm 0.28 (4). Regarding the barrier properties, the solubility values were lower in those containing chitosan due to their insolubility in water, $22 \%$ for 1 and $18 \%$ for 5 . The films formed by the blends provided a reduction in the water permeation rate without compromise its characteristics, such as 4 and 5, with a rate of $14.15 \mathrm{~g} \cdot \mathrm{m}^{-2} \cdot \mathrm{h}^{-1}$ and $20.43 \mathrm{~g} \cdot \mathrm{m}^{-2} \cdot \mathrm{h}^{-1}$, respectively.

facilmente. Para tentar sanar esse problema, estão ganhando destaques novos estudos com a mistura entre os polímeros, as chamadas blendas poliméricas, com a finalidade de formar novos materiais com propriedades de superfície melhoradas e biocompatibilidade em comparação com as do único componente (LEWANDOWSKA et al., 2015).

\section{Revista Verde \\ ISSN 1981-8203}

Pombal, Paraíba, Brasil v. 15, n.4, out.-dez., p. 391-397, 2020 doi: $10.18378 /$ rvads.v15i4.6713 
Para a formação de blendas poliméricas, os polissacarídeos estão sendo estudados como bons candidatos, pois são amplamente utilizados para a obtenção de filmes comestíveis, devido às suas propriedades e estruturas de redes poliméricas, e principalmente por serem boas barreiras ao dióxido de carbono, eficazes na redução das trocas gasosas e das taxas de reações oxidativas, no entanto, apresentam a desvantagem de serem bastante hidrofílicos (ALVES et al., 2010).

A quitosana é um polissacarídeo derivado da desacetilação da quitina com características de biodegradabilidade, antimicrobiana e antifúngica (THEVARAJAH et al., 2017). Entretanto, tem alta permeabilidade ao vapor de água, principalmente devido ao seu caráter hidrofílico (AGUIRRE-LOREDO et al., 2016).

A pectina é um polissacarídeo natural e a sua utilização é crescente devido à elevada disponibilidade e à não toxicidade, aliadas ao baixo custo (RAMPINO et al., 2016), porém, como desvantagem apresentam filmes muito frágeis e hidrofílicos, o que impede sua ampla aplicação (NESIC et al., 2017).

A fécula de mandioca é um polímero natural obtido de recursos renováveis, abundante e de baixo custo, com comportamento termoplástico (MARAN et al., 2013; SELIGRA et al., 2016). No entanto, filmes obtidos a partir desse material possuem restrições tecnológicas devido ao seu caráter hidrofílico e à sua alta retrogradação. A partir disso, a reação de reticulação com ácido cítrico é comum para limitar a retrogradação do amido e melhorar o seu desempenho para várias aplicações (SELIGRA et al., 2016).

Baseado no exposto objetivou-se desenvolver filmes comestíveis produzidos por blendas poliméricas dos polissacarídeos naturais, quitosana, pectina e fécula de mandioca.

\section{MATERIAL E MÉTODOS}

Os materiais utilizados foram: quitosana em pó (Grau de desacetilação de 85\%), fornecida pela Polymar Indústria e Com. Imp. e Exp Ltda® - Brasil; amido de mandioca (18\% em peso de amilose e $82 \%$ em peso de amilopectina), obtido da Primícias do Brasil ${ }^{\circledR}$, localizada na cidade de Macaíba - RN; pectina e glicerol, obtidos da Dinâmica Química Contemporânea Ltda - Brasil; ácido acético P.A glacial, pureza mínima de 99,8\%, obtido da PROQUíMIOS Comércio e Indústria ${ }^{\circledR}$, localizada no Rio de Janeiro - Brasil; ácido cítrico anidro P.A, usado como agente de reticulação do amido, obtido da QHEMIS Indústria química do Brasil Ltda ${ }^{\circledR}-$ Brasil.

Foram preparadas soluções individuais de cada componente polimérico, a uma concentração de $2 \%$ em relação à massa seca do polímero $20 \mathrm{~g} / \mathrm{L}$, e o glicerol, adicionado em $20 \%$ (base seca de polímero), como plastificante. A solução de quitosana foi preparada por dissolução em ácido acético $1 \%$, sob agitação para a completa dissolução por até $12 \mathrm{~h}$ a $26^{\circ} \mathrm{C}$ (BARON et al., 2017). A solução de fécula de mandioca foi preparada por dissolução do polímero em água destilada. Ácido cítrico foi adicionado a 10\% (base seca de fécula) como agente reticulante, conforme descrito por Seligra et al., (2016); a mistura foi agitada até completa dissolução sob aquecimento até $70^{\circ} \mathrm{C}$; atingindo essa temperatura, a agitação continuou por mais 15 minutos. A solução de pectina foi preparada diluindo o polímero em água destilada, com agitação constante até a completa dissolução (BARON et al., 2017).
As soluções foram depositadas $(80 \mathrm{~mL})$ em placas de acrílico com diâmetro de $15 \mathrm{~cm}$. Os filmes foram formados pela técnica casting, sendo deixados para secar durante 4 à $6 \mathrm{~h}$, utilizando uma estufa com circulação de ar a $40^{\circ} \mathrm{C}$. A Tabela 1 resume a constituição de cada filme.

Tabela 1. Planejamento Experimental com a composição dos filmes comestíveis produzidos

\begin{tabular}{llcc}
\hline Filme & Biopolímero & Composição (\%) \\
\hline 1 & Quitosana & 100 \\
2 & Pectina & 100 \\
3 & Fécula de Mandioca & 100 \\
4 & Quitosana + Pectina & $50+50$ \\
5 & Quitosana + Fécula de & $50+50$ \\
& Mandioca & & $50+50$ \\
6 & Pectina + Fécula de & \\
& Mandioca & Quitosana + Pectina + & $33,33+33,33+$ \\
7 & Fécula de Mandioca & 33,33 \\
\hline
\end{tabular}

A espessura média foi realizada por um micrômetro digital Mitutoyo ${ }^{\circledR}$ (precisão de $0,001 \mathrm{~mm}$ ) em 5 posições diferentes dos filmes, de acordo com Luchese et al., (2015). As medidas de cor dos filmes, assim como a opacidade, foram determinadas com um colorímetro Konica Minolta ${ }^{\circledR}$, modelo CR-10, e utilizando os parâmetros de cores CIELab de acordo com Zavareze et al., (2012). Avaliaram-se os valores de L [luminosidade (0 - preto, 100 - branco)], a [de verde (-) ao vermelho (+)], e b [de azul (-) a amarelo (+)].

A opacidade dos filmes (Y) foi realizada de acordo com o método de Fakhouri et al., (2015), sendo calculada conforme Eq. 1, descrita por Zavareze et al., (2012), sendo $Y_{\text {preto }}$ e $Y_{\text {branco }}$ a opacidade obtida em fundo preto e em fundo branco, respectivamente.

$$
\mathrm{Y}=\left(\mathrm{Y}_{\text {preto }} / \mathrm{Y}_{\text {branco }}\right) \times 100
$$

A determinação da solubilidade (S) foi seguida de acordo com metodologia desenvolvida por Casariego et al. (2009), e determinada conforme a Eq. 2, onde $\mathrm{m}_{\mathrm{i}}$ e $\mathrm{m}_{\mathrm{f}}$ são as massas inicial e final do filme:

$$
\mathrm{S}=\left(\left(\mathrm{m}_{\mathrm{i}}-\mathrm{m}_{\mathrm{f}}\right) / \mathrm{m}_{\mathrm{i}}\right) \times 100
$$

A Taxa de Permeabilidade ao Vapor de Água (TPVA) dos filmes foi determinada utilizando adaptação da norma padrão ASTM E96-80 da American Society for Testing and Materials (ASTM, 1980). Foram cortados quadrados de $2 \mathrm{~cm} \mathrm{x} 2 \mathrm{~cm}$ e posicionados sobre o orifício de células de permeação, de modo que a única perda de massa ocorre pelo fluxo de vapor de água através dos filmes. Em seguida as células foram depositadas dentro de um dessecador contendo sílica como dessecante. Foram realizadas pesagens de hora em hora, medindo a massa das células de permeabilidade. Foi calculada e plotada a inclinação da curva (massa de água versus tempo) por regressão linear, representada pelas alterações no peso de água que se difunde através do filme por unidade de tempo (g/s). A TPVA foi calculada a partir do coeficiente linear da reta dividido pela área de transferência $\left(\mathrm{m}^{2}\right)$. Assim, a TPVA $\left(\mathrm{g} / \mathrm{m}^{2} . \mathrm{h}\right)$ dos filmes foi medida de acordo com a Eq. 3 .

$$
\mathrm{TPVA}=\mathrm{m} /(\mathrm{t} \times \mathrm{A})
$$

Em que: $\mathrm{m}$ - massa de água que atravessa o filme $(\mathrm{g}) ; \mathrm{t}$ - tempo no qual ocorre a perda de massa (h); A - área exposta do filme $\left(\mathrm{m}^{2}\right)$. 
O ângulo de contato foi determinado pela técnica da gota séssil, composto por uma base móvel, uma câmera (VP 540s, Intelbras ${ }^{\circledR}$ ) e uma pipeta (PASSERONE et al., 1979; SIDDIQI et al., 2000; BOINOVICH et al., 2014). A resistência à tração e a percentagem de alongamento à ruptura foram avaliadas por um teste de tração segundo o método ASTM D-882-91 (1996), utilizando-se uma máquina universal de ensaios DL 10000, com capacidade máxima de $100 \mathrm{kN}$, marca EMIC Equipamentos e Sistemas de Ensaios Ltda ${ }^{\circledR}$.

As amostras seguiram a mesma norma e foram avaliadas com $50 \mathrm{~mm}$ de comprimento e $5 \mathrm{~mm}$ de largura. A força e a distância foram registradas durante a extensão das amostras a $0,8 \mathrm{~mm} / \mathrm{s}$ até à ruptura. A resistência à tração foi calculada dividindo a força máxima pela secção transversal do filme, conforme a Eq.4. O alongamento na ruptura foi calculado pelo comprimento causado pela tensão dividido pelo comprimento inicial, de acordo com a Eq. 5.

$$
\begin{aligned}
& \sigma=\mathrm{F}_{\mathrm{m}} / \mathrm{A} \\
& \epsilon=\Delta \mathrm{L} / \mathrm{L}_{0} \times 100
\end{aligned}
$$

Em que: $\sigma$ - resistência à tração $(\mathrm{MPa}) ; \mathrm{F}_{\mathrm{m}}$ - força máxima $(\mathrm{N})$; A - área de seção transversal do filme $\left(\mathrm{m}^{2}\right) ; \epsilon$ - alongamento (\%); $\mathrm{L}_{0}$ - distância inicial de separação (m); L - distância de ruptura $(\mathrm{m})$.

A avaliação da morfologia dos filmes foi feita por microscópio eletrônico de varredura, com alta resolução da superfície das amostras. As amostras de filme foram fixadas em suportes (stubs) de alumínio, com fita adesiva de carbono e recobertas com uma fina camada de ouro, em um metalizador a vácuo, modelo Q150R. Foi aplicada uma tensão de $10 \mathrm{kV}$, com magnitude de 1000x. O equipamento utilizado é da marca TESCAN $^{\circledR}$, modelo VEGA 3.

Todos os ensaios foram realizados em triplicata. Os dados foram submetidos à análise de variância (ANOVA), e as médias comparadas pelo teste de Tukey a 5\% de probabilidade. O software utilizado foi o Sttatistica versão 13.2 (Tibco Inc., USA).

\section{RESULTADOS E DISCUSSÃO}

A Tabela 2 apresenta os valores de espessura, parâmetros de cor e opacidade dos filmes e blendas biopoliméricas. Houve diferença estatística $(p<0,05)$ para a espessura comparando as blendas com os filmes individuais. A variação nos valores das espessuras dos filmes é decorrente principalmente do processo casting, onde os filmes são secos em suportes, gerando pequenas variações de espessuras. Bof et al., (2015) encontraram cerca de $0,066 \mathrm{~mm}$ para blendas de amido de milho e quitosana (na proporção de $50 \%$ de cada polímero), havendo diferença estatística $(\mathrm{p}<0,05)$ comparando as blendas com os filmes individuais.

Tabela 2. Espessura e propriedades ópticas dos filmes comestíveis produzidos por blendas poliméricas dos polissacarídeos naturais, quitosana, pectina e fécula de mandioca

\begin{tabular}{cccccc}
\hline \multirow{2}{*}{ Filme } & Espessura $(\mathrm{mm})$ & \multicolumn{3}{c}{ COR } & Opacidade \\
\cline { 3 - 5 } & $0,059 \pm 0,002^{\mathrm{cd}}$ & $-3,55 \pm 0,07^{\mathrm{c}}$ & $34,65 \pm 1,91^{\mathrm{a}}$ & $81,00 \pm 0,71^{\mathrm{b}}$ & $45,31 \pm 0,13^{\mathrm{b}}$ \\
\hline 1 & $0,055 \pm 0,004^{\mathrm{de}}$ & $-2,95 \pm 0,07^{\mathrm{d}}$ & $12,15 \pm 0,21^{\mathrm{b}}$ & $83,15 \pm 0,07^{\mathrm{cd}}$ & $45,40 \pm 0,12^{\mathrm{b}}$ \\
3 & $0,050 \pm 0,001^{\mathrm{e}}$ & $-2,85 \pm 0,07^{\mathrm{d}}$ & $7,85 \pm 0,07^{\mathrm{b}}$ & $84,40 \pm 0,14^{\mathrm{c}}$ & $45,14 \pm 0,24^{\mathrm{b}}$ \\
4 & $0,067 \pm 0,004^{\mathrm{a}}$ & $-3,05 \pm 0,21^{\mathrm{d}}$ & $33,70 \pm 1,98^{\mathrm{a}}$ & $77,40 \pm 0,28^{\mathrm{a}}$ & $46,96 \pm 0,45^{\mathrm{a}}$ \\
5 & $0,056 \pm 0,002^{\mathrm{cd}}$ & $-3,85 \pm 0,07^{\mathrm{c}}$ & $17,00 \pm 0,14^{\mathrm{c}}$ & $82,20 \pm 0,14^{\text {bd }}$ & $45,56 \pm 0,16^{\mathrm{b}}$ \\
6 & $0,041 \pm 0,002^{\mathrm{b}}$ & $-7,65 \pm 0,07^{\mathrm{a}}$ & $10,55 \pm 0,21^{\mathrm{b}}$ & $83,65 \pm 0,07^{\mathrm{cd}}$ & $45,31 \pm 0,13^{\mathrm{b}}$ \\
7 & $0,061 \pm 0,003^{\mathrm{c}}$ & $-4,95 \pm 0,07^{\mathrm{b}}$ & $20,05 \pm 1,48^{\mathrm{c}}$ & $81,20 \pm 0,57^{\mathrm{b}}$ & $45,75 \pm 0,58^{\mathrm{ab}}$ \\
\hline
\end{tabular}

Letras diferentes nas colunas indicam diferença estatística (p<0,05), pelo teste de Tukey a 5\% de significância. Filme 1 - $100 \%$ quitosana; Filme 2 - $100 \%$ pectina; Filme $3-100 \%$ fécula de mandioca; Filme $4-50 \%$ quitosana $+50 \%$ pectina; Filme $5-50 \%$ quitosana $+50 \%$ fécula de mandioca; Filme $6-50 \%$ pectina $+50 \%$ fécula de mandioca; Filme $7-33,33 \%$ quitosana $+33,33 \%$ pectina $+33,34 \%$ fécula de mandioca

Azevedo et al. (2017) estudando o efeito da substituição do amido de milho por proteína de soro de leite em blendas biodegradáveis, encontraram filmes mais espessos variando entre 0,33 e $0,39 \mathrm{~mm}$ e não apresentaram diferença significativa ( $p<0,05)$. Fakhoury et al. (2012) formulando filmes a partir de misturas de amido de mandioca e gelatina com diferentes plastificantes, obtiveram filmes transparentes e ligeiramente brilhantes com espessura variando entre 0,034 e $0,075 \mathrm{~mm}$, percebendo aumento na espessura a medida que aumentava a quantidade de amido ou gelatina.

$\mathrm{O}$ parâmetro $\mathrm{a}^{*}$, que corresponde a uma tendência ao verde, apresentou diferença significativa $(\mathrm{p}<0,05)$ para as blendas. Os filmes contendo quitosana apresentam uma coloração mais amarelada, parâmetro b*, mostrando diferença significativa $(\mathrm{p}<0,05)$. $\mathrm{O}$ parâmetro $\mathrm{L}^{*}$, referente à luminosidade, sofreu uma redução para os filmes 1,5 e 7 . Todos estes resultados estão associados à cor natural da matéria-prima utilizada para obtenção dos mesmos (o pó de quitosana).
A opacidade dos filmes diferiu estatisticamente $(\mathrm{p}<0,05)$ apenas para o filme 4 , havendo uma redução da luminosidade $\mathrm{L}^{*}=77,40$, corroborando com o valor encontrado de opacidade para este filme $(46,96)$.

Azevedo et al. (2017) estudando as propriedades de transparência óptica em filmes com substituição do amido de milho pelo isolado de proteína de soro de leite, concluíram que estas apresentaram diferença significativa $(p<0,05)$. Para a luminosidade $\left(\mathrm{L}^{*}\right)$, não apresentou diferença significativa $(\mathrm{p}$ $<0,05)$ variando de 85,17 a 88,75 .

A Tabela 3 apresenta os resultados de solubilidade, TPVA, ângulo de contato e as medidas de resistência à tração e alongamento na ruptura dos filmes e blendas poliméricas. Os filmes 2 e 6 se dispersaram e perderam sua integridade, devido à alta solubilidade em água, o que não é uma característica desejada para filmes que serão usados para o recobrimento de frutos, pois estes não devem se desintegrar tão facilmente. Uma diferença estatística $(\mathrm{p}<0,05)$ foi observada entre a solubilidade das blendas em comparação com os filmes individuais. 
Tabela 3. Propriedades de barreira, ângulo de contato $(\theta)$ e mecânicas dos filmes comestíveis produzidos por blendas poliméricas dos polissacarídeos naturais, quitosana, pectina e fécula de mandioca

\begin{tabular}{cccccc}
\hline Filme & Solubilidade $(\%)$ & TPVA $\left(\mathrm{g} \cdot \mathrm{m}^{-2} \cdot \mathrm{h}^{-1}\right)$ & $\theta\left(^{\circ}\right)$ & $\sigma(\mathrm{MPa})$ & $\epsilon(\%)$ \\
\hline 1 & $22,34 \pm 0,94^{\mathrm{bd}}$ & $121,82 \pm 0,07^{\mathrm{b}}$ & $56,20 \pm 1,55^{\mathrm{c}}$ & $0,032 \pm 0,003^{\mathrm{b}}$ & $3,154 \pm 0,137^{\mathrm{a}}$ \\
2 & $64,08 \pm 1,53^{\mathrm{a}}$ & $99,42 \pm 0,05^{\mathrm{a}}$ & $39,89 \pm 1,29^{\mathrm{a}}$ & $0,018 \pm 0,004^{\mathrm{ce}}$ & $2,536 \pm 0,484^{\mathrm{ab}}$ \\
3 & $30,36 \pm 0,37^{\mathrm{c}}$ & $100,99 \pm 0,06^{\mathrm{a}}$ & $36,22 \pm 2,68^{\mathrm{a}}$ & $0,004 \pm 0,003^{\mathrm{a}}$ & $1,540 \pm 0,660^{\mathrm{b}}$ \\
4 & $26,31 \pm 1,85^{\mathrm{bc}}$ & $14,15 \pm 0,01^{\mathrm{c}}$ & $60,84 \pm 0,46^{\mathrm{c}}$ & $0,014 \pm 0,005^{\mathrm{e}}$ & $1,816 \pm 0,624^{\mathrm{b}}$ \\
5 & $18,14 \pm 1,61^{\mathrm{d}}$ & $20,43 \pm 0,01^{\mathrm{d}}$ & $49,02 \pm 2,64^{\mathrm{b}}$ & $0,031 \pm 0,003^{\mathrm{bd}}$ & $2,402 \pm 0,260^{\mathrm{ab}}$ \\
6 & $65,03 \pm 0,04^{\mathrm{a}}$ & $61,70 \pm 0,03^{\mathrm{e}}$ & $59,79 \pm 2,12^{\mathrm{c}}$ & $0,013 \pm 0,003^{\mathrm{e}}$ & $1,692 \pm 0,559^{\mathrm{b}}$ \\
7 & $24,66 \pm 1,68^{\mathrm{b}}$ & $81,08 \pm 0,05^{\mathrm{f}}$ & $60,80 \pm 1,59^{\mathrm{c}}$ & $0,023 \pm 0,001^{\mathrm{cd}}$ & $1,703 \pm 0,079^{\mathrm{b}}$ \\
\hline
\end{tabular}

Letras diferentes nas colunas indicam diferença estatística ( $<<0,05)$, pelo teste de Tukey a $5 \%$ de significância. TPVA - taxa de permeação ao vapor de água, $\theta$ - ângulo de contato, $\sigma$ - resistência à tração, $\epsilon$ - alongamento na ruptura. Filme 1 - 100\% quitosana; Filme 2 - $100 \%$ pectina; Filme 3 - $100 \%$ fécula de mandioca; Filme $4-50 \%$ quitosana $+50 \%$ pectina; Filme $5-50 \%$ quitosana $+50 \%$ fécula de mandioca; Filme $6-50 \%$ pectina $+50 \%$ fécula de mandioca; Filme $7-$ $33,33 \%$ quitosana $+33,33 \%$ pectina $+33,34 \%$ fécula de mandioca

Observou-se uma discreta diminuição dos valores de solubilidade nas misturas binárias e na ternária com exceção do filme 6 que se manteve na média de $65 \%$ de solubilidade em água. Isso se deve a interação da hidroxila do glicerol com a matriz polimérica dos polissacarídeos, aumentando os espaços entre as cadeias e a mobilidade molecular, facilitando a migração de moléculas de vapor de água pelo filme e, por conseguinte, aumentando a solubilidade (BELIBI et al., 2014).

Os valores mais altos de TPVA nos filmes individuais podem ser explicados pela interação com o glicerol, onde este pode alojar-se facilmente entre as moléculas do polímero e formar ligações de hidrogênio com os grupos hidroxila (AZEVEDO et al., 2017). O comportamento observado para as blendas demonstra que a integridade destas foi $o$ fator determinante para suas propriedades de barreira, e pode ser explicado pela teoria de volume livre para polímeros, em que um plastificante a baixa concentração é intercalado na cadeia polimérica sem levar a um aumento no volume livre (MENEGUIN et al., 2014). Com ênfase para o filme 5, da blenda quitosana/fécula, onde o tipo de ligação de hidrogênio reduz a disponibilidade de grupos hidrofílicos nas matrizes de quitosana, reduzindo a TPVA (BOF et al., 2015).

Chillo et al. (2008) observaram que a TPVA é influenciada pelo glicerol, sendo maior à medida que aumenta a concentração do plastificante.

Azevedo et al. (2015) estudaram o efeito do ácido cítrico em filmes de proteína de soro de leite e relataram uma redução na TPVA com adição de ácido cítrico a 5\%. Segundo os autores, a ligação de hidrogênio formada com um grupo hidroxila e um grupo carboxila aumenta a interação inter/intramolecular entre os aditivos presentes no filme.

Levando em consideração o que Jaramillo et al. (2015) propõem em seus estudos, pode-se admitir que a menor hidrofilia ou maior hidrofobicidade será encontrada em ângulos acima de $65^{\circ}$ e o contrário se admite para ângulos abaixo de $65^{\circ}$.

Jaramillo et al., (2015) e Hosseini et al., (2016) afirmam que um aumento dos valores de ângulo de contato das blendas, demonstra que estas apresentam maior hidrofobicidade em comparação com os filmes individuais. Assim, os valores de ângulo de contato encontrados demonstraram filmes hidrofílicos, com resultados que também sugerem que os materiais produzidos não oferecem uma barreira superficial significativa e que as forças adesivas são maiores em relação às forças coesivas da água (JARAMILLO et al., 2015).

Na Tabela 3 estão também os valores das propriedades mecânicas dos filmes de resistência à tração e alongamento na ruptura. Os resultados dos parâmetros de tração apresentam valores de rigidez com uma leve alteração principalmente nas blendas que continham fécula de mandioca (filme $5 \mathrm{com} \sigma=$ $0,031 \mathrm{MPa}$ e filme $7 \mathrm{com} \sigma=0,023 \mathrm{MPa}$ ) em comparação com o filme individual deste polímero que apresentava $\sigma=0,004$ $\mathrm{MPa}$. O alongamento na ruptura não apresentou diferença significativa $(\mathrm{p}<0,05)$, com a mistura de polímeros, apenas no filme 5, blenda de quitosana e fécula houve um aumento do alongamento para $\epsilon=2,402 \%$ em relação ao filme individual de fécula de mandioca. Estas características são importantes nos materiais de embalagem e podem contribuir para prever suas propriedades mecânicas como embalagens de alimentos (SONG et al., 2018).

A Figura 1 apresenta imagens da análise morfológica realizada dos filmes. Nas Figuras 1A e 1B, observa-se uma estrutura coesa e sem poros, indicando gelatinização eficiente. No filme de fécula, Figura $1 \mathrm{C}$, percebe-se o grau de rugosidade alterado, porém diminuído nas blendas que continham esse polímero, podendo-se inferir que isso tenha sido ocasionado pela adição do ácido cítrico nessa solução polimérica. Bonilla et al. (2013) estudando as propriedades da blenda de amido de trigo e quitosana com a incorporação de antioxidantes, obtiveram uma microestrutura de filme pouco heterogêneo inferindo que o ácido cítrico não afetou a rugosidade. Nos filmes 4 e 7, Figuras 1D e 1G, ocorre uma certa irregularidade, como um emaranhado na estrutura, ocasionada por uma possível separação de fases, podendo indicar que são misturas parcialmente miscíveis. Essas irregularidades podem ter ocorrido devido à não uniformização das soluções filmogênicas; ou pela presença de mais de uma macromolécula na matriz polimérica; ou ainda, as mudanças observadas na morfologia estão relacionadas às interações ente os compostos poliméricos onde as forças repulsivas e/ou interações eletrostáticas entre os componentes nas misturas podem levar ao aumento de tamanhos de microdomínios (LEWANDOWSKA et al., 2015). 
Figura 1. Microscopia eletrônica de varredura dos filmes: Quitosana (A), Pectina (B), Fécula de Mandioca (C), Quitosana/Pectina (D), Quitosana/Fécula (E), Pectina/Fécula (F), Quitosana/pectina/fécula (G).

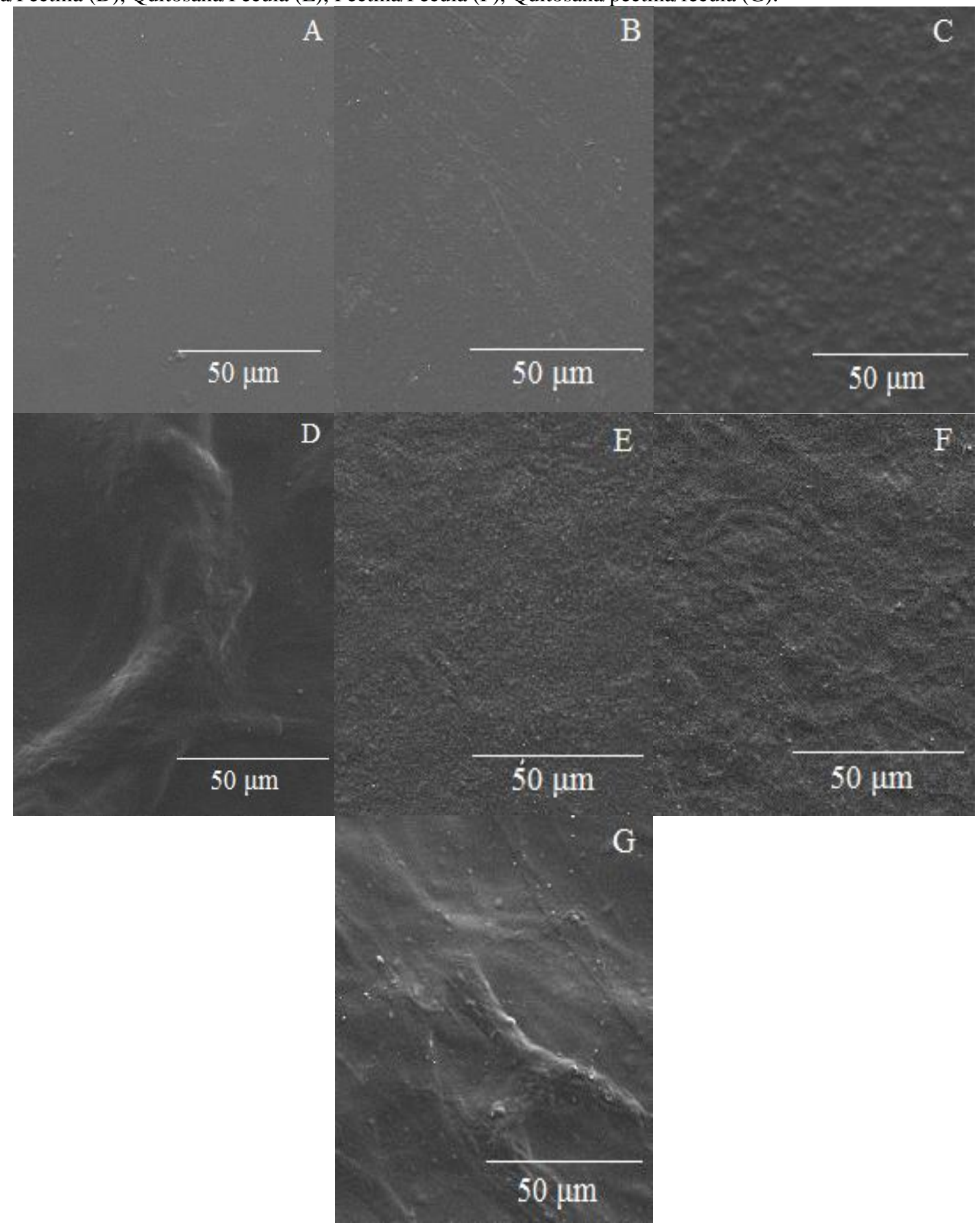

\section{CONCLUSÕES}

A melhor blenda é a de quitosana/fécula de mandioca, pois obteve bom desempenho com relação às propriedades de solubilidade, TPVA, e a miscibilidade. Sendo assim, a formação de blendas modifica a estrutura polimérica dos filmes puros, proporcionando melhoras nas propriedades dos filmes poliméricos.

\section{AGRADECIMENTOS}

Os autores agradecem a Universidade Federal Rural do Semi-Árido, por todo suporte ao trabalho.

\section{REFERÊNCIAS}

AGUIRRE - LOREDO, R. Y.; RODRÍGUEZ HERNÁNDEZ, A. I.; MORALES - SÁNCHEZ, E.; GÓMEZALDAPA, C. A.; VELAZQUEZ, G. Effect of equilibrium moisture content on barrier, mechanical and thermal properties of chitosan films. Food Chemistry, v. 196, p. 560-566, 2016. $\underline{10.1016 / \text { j.foodchem.2015.09.065 }}$

ALVES, V. D.; COSTA, N.; COElHOSO, I. M. Barrier properties of biodegradable composite films based on kappacarrageenan/pectin blends and mica flakes. Carbohydrate 
Polymers, v. 79, n. 2, p. 269-276, 2010 $\underline{10.1016 / \text { j.carbpol.2009.08.002 }}$

ASTM E96/E80M (1980). Standard test methods for water vapour transmission of materials. Annual Book of Standards.

ASTM D882-91. (1996). Standard test methods for tensile properties of thin plastic sheeting. Annual Book of Standards.

AZEVEDO, V. M.; SILVA, E. K.; PEREIRA, C. F. G.; COSTA, J. M. G. DA; BORGES, S. V. Whey protein isolate biodegradable films: Influence of the citric acid and montmorillonite clay nanoparticles on the physical properties. Food Hydrocolloids, v. 43, p. 252-258, 2015. $\underline{10.1016 / \text { j.foodhyd.2014.05.027 }}$

AZEVEDO, V. M.; BORGES, S. V.; MARCONCINI, J. M.; YOSHIDA, M. I.; NETO, A. R. S.; PEREIRA, T. C.; PEREIRA, C. F. G. Effect of replacement of corn starch by whey protein isolate in biodegradable film blends obtained by extrusion. Carbohydrate Polymers, v. 157, p. 971-980, 2017. $\underline{10.1016 / \text { j.carbpol.2016.10.046 }}$

BARON, R. D.; PÉREZ, L. L.; SALCEDO, J. M.; CÓRDOBA, L. P.; SOBRAL, P. J. DO A. Production and characterization of films based on blends of chitosan from blue crab (Callinectes sapidus) waste and pectin from Orange (Citrus sinensis Osbeck) peel. International Journal of Biological Macromolecules, v. 98, p. 676-683, 2017. 10.1016/j.ijbiomac.2017.02.004.

BELIBI, P. C.; DAOU, T. J.; NDJAKA, J. M. B.; NSOM, D.; MICHELIN, L.; DURAND, B. A comparative study of some properties of cassava and tree cassava starch films. Physics Procedia, v. 55, p. 220-226, 2014. 10.1016/j.phpro.2014.07.032 .

BOF, M. J.; BORDAGARAY, V. C.; LOCASO, D. E.; GARCÍA, M. A. Chitosan molecular weight effect on starchcomposite film properties. Food Hydrocolloids, v. 51, p. $281-$ 294, 2015. 10.1016/j.foodhyd.2015.05.018

BOINOVICH, L.; EMELYANENKO, A. M.; KOROLEV, V. V; PASHININ, A. S. Effect of wettability on sessile drop freezing: when superhydrophobicity stimulates an extreme freezing delay. Langmuir, v. 30, n. 6, p. 1659-1668 , 2014. $\underline{10.1021 / 1 \mathrm{a} 403796 \mathrm{~g}}$

BONILLA, J.; TALÓN, E.; ATARÉS, L.; VARGAS, M.; CHIRALT, A. Effect of the incorporation of antioxidants on physicochemical and antioxidant properties of wheat starch chitosan films. Journal of Food Engineering, v. 118, n. 3, p. 271-278, 2013. 10.1016/j.jfoodeng.2013.04.008.

CASARIEGO, A.; SOUZA, B. W. S.; CERQUEIRA, M. A.; TEIXEIRA, J. A.; CRUS, L.; DÍAZ, R.; VICENTE, A. A. Chitosan/clay films properties as affected by biopolymer and clay micro/nanoparticles concentrations. Food Hydrocolloids, v. 23, n. 7, p. 1895-1902, 2009. $\underline{10.1016 / \text { j.foodhyd.2009.02.007 }}$

CHILLO, S.; FLORES, S.; MASTROMATTEO, M.; CONTE, A.; GERSCHENSON, L.; DEL NOBILE, M. A. Influence of glycerol and chitosan on tapioca starch-based edible film properties. Journal of Food Engineering, v. 88, n. 2, p. 159168, 2008. 10.1016/j.jfoodeng.2008.02.002

CIELAB - COMISSÃO INTERNACIONAL DE ILUMINAÇÃO - Sistema de cores, 1976. Disponível em: <http://www.sightgrip.co.uk/bbstest.htm>.

FAKHOURI, F. M.; MARTELLI, S. M.; CAON, T.; VELASCO, J. I.; MEI, L. H. I. Edible films and coatings based on starch/gelatin: Film properties and effect of coatings on quality of refrigerated red crimson grapes. Postharvest Biology and Technology, v. 109, p. 57-64, 2015. 10.1016/j.postharvbio.2015.05.015.

HOSSEINI, S. F.; REZAEI, M.; ZANDI, M.; FARAHMANDGHAVI, F. Development of bioactive fish gelatin/chitosan nanoparticles composite films with antimicrobial properties. Food Chemistry, v. 194, p. 12661274, 2016. 10.1016/j.foodchem.2015.09.004.

JARAMILLO, C. M.; SELIGRA, P. G.; GOYANES, S.; BERNAL, C.; FAMÁ, L. Biofilms based on cassava starch containing extract of yerba mate as antioxidant and plasticizer. Starch, v. 67, p. 780-789, 2015. 10.1002/star.201500033.

LEWANDOWSKA, K.; SIONKOWSKA, A.; GRABSKA, S. Chitosan blends containing hyaluronic acid and collagen. Compatibility behaviour. Journal of Molecular Liquids, v. 212, p. 879-884, 2015. 10.1016/j.molliq.2015.10.047.

LUCHESE, C. L.; FRICK, J. M.; PATZER, V. L.; SPADA, J. C.; TESSARO, I. C. Synthesis and characterization of biofilms using native and modified pinhão starch. Food Hydrocolloids, v. 45, p. 203-210, 2015. 10.1016/j.foodhyd.2014.11.015

MARAN, J. P.; SIVAKUMAR, V.; SRIDHAR, R.; THIRUGNANASAMBANDHAM, K. Development of model for barrier and optical properties of tapioca starch based edible films. Carbohydrate Polymers, v. 92, n. 2, p. 1335-1347, 2013. $\underline{10.1016 / j . c a r b p o l .2012 .09 .069}$.

MENEGUIN, A. B.; CURY, B. S. F.; EVANGELISTA, R. C. Films from resistant starch-pectin dispersions intended for colonic drug delivery. Carbohydrate Polymers, v. 99, p. 140149, 2014. 10.1016/j.carbpol.2013.07.077.

NESIC, A.; ONJIA, A.; DAVIDOVIC, S.; DIMITRIJEVIC, S; ERRICO, M. E.; SANTAGATA, G.; MALINCONICO, M. Design of pectin-sodium alginate based films for potential healthcare application: Study of chemico-physical interactions between the components of films and assessment of their antimicrobial activity. Carbohydrate Polymers, v. 157, p. 981990, 2017. 10.1016/j.carbpol.2016.10.054

PASSERONE, A.; SANGIORGI, R.; VALBUSA, G. surface tension and density of molten glasses in the system $\mathrm{La}_{2} \mathrm{O}_{3} \mathrm{Na}_{2}$ $\mathrm{Si}_{2} \mathrm{O}_{5}$. Ceramurgia International, v. 5. n. 1. p. 18-22, 1979. 10.1016/0390-5519(79)90005-X.

RAMPINO, A.; BORGOGNA, M.; BELLICH, B.; BLASI, P.; VIRGILIO, F.; CESARO, A. Chitosan-pectin hybrid nanoparticles prepared by coating and blending techniques. 
European Journal of Pharmaceutical Sciences, v. 84, p. 37-45, 2016. 10.1016/j.ejps.2016.01.004.

SELIGRA, P. G.; JARAMILLO, C. M.; FAMÁ, L.; GOYANES, S. Biodegradable and non-retrogradable ecofilms based on starch-glycerol with citric acid as crosslinking agent. Carbohydrate Polymers, v. 138, p. 66-74, 2016. 10.1016/j.carbpol.2015.11.041.

SIDDIQI, N.; BHOI, B.; PARAMGURU, R. K.; SAHAJWALLA, V.; OSTROVSKI, O. Slag - graphite wettability and reaction kinetics Part 1 Kinetics and mechanism of molten $\mathrm{FeO}$ reduction reaction. Ironmaking \& steelmaking, v. $27, \quad$ n. $5, \quad$ p. 367-372, 2000. $\underline{10.1179 / 030192300677679}$.

SONG, X.; ZUO, G.; CHEN, F. Effect of essential oil and surfactant on the physical and antimicrobial properties of corn and wheat starch films. International Journal of Biological Macromolecules, v. 107, p. 1302-1309, 2018 $\underline{10.1016 / j . i j b i o m a c .2017 .09 .114 . ~}$

THEVARAJAH, J. J.; LEEUWEN, M. P. VAN; COTTET, H.; CASTIGNOLLES, P.; GABORIEAU, M. Determination of the distributions of degrees of acetylation of chitosan. International Journal of Biological Macromolecules, v. 95, p. 40-48, 2017. 10.1016/j.ijbiomac.2016.10.056.

ZAVAREZE, E. DA R.; PINTO, V. Z.; KLEIN, B.; HALAL, S. M. L. EL; ELIAS, M. C.; HERNÁNDEZ, C. P.; DIAS, A. R. G. Development of oxidised and heat-moisture treated potato starch film. Food chemistry, v. 132, n. 1, p. 344-350, 2012. 10.1016/j.foodchem.2011.10.090. 